\title{
LA EDUCACIÓN PARA LA SALUD EN LOS MANUALES ESCOLARES. EJEMPLOS CONCRETOS EN TORNO A 1930-1950
}

\author{
Ma Ángeles Valdemoros San Emeterio \\ Jesús Ramírez Martínez \\ Universidad de La Rioja \\ Juan Carlos Martínez-Iñiguez \\ Servicio Riojano de Salud (SERIS)
}

\begin{abstract}
RESUMEN: Con este trabajo se pretende analizar el tratamiento de la educación para la salud en los manuales escolares del periodo 1930-1950, y comparar el mismo con el que se lleva a cabo en la actualidad. Se intentará comprobar que muchos conceptos e ideas actuales ya estaban presentes en aquellas décadas. Asimismo, se destacarán las diferencias, especialmente pedagógicas y didácticas, encontradas en la forma de llevar a cabo la educación para la salud antes y ahora. Se hará desde una doble perspectiva: 1. Concepto de salud, de higiene y de la pedagogía de educación para la salud. 2. Programa de contenidos de educación para la salud en esos materiales didácticos.
\end{abstract}

ABSTRACT: This work aims to analyze the treatment of health education in school textbooks during the period 1930-1950, and to compare it with the one that is conducted at present. It will attempt to verify how many current concepts and ideas were already present in those decades. In addition, the differences in the way of carrying out health education then and now will be outlined, especially those referred to pedagogic strategies and didactic materials. All this will be done from a double perspective: 1 . The concept of health, hygiene and pedagogy of health education. 2. The program contents of health education in the didactic materials.

PALABRAS CLAVE: Educación para la salud, manuales escolares, materiales didácticos, salud, higiene, multiculturalidad e interculturalidad, análisis comparativo.

KEYWORDS: Health education, textbooks, didactic materials, health, hygiene, multiculturalism, intercultural, comparative analysis. 


\section{INTRODUCCIÓN}

Este trabajo se constituye en una continuidad de diversas experiencias, estudios y revisiones llevados a cabo con anterioridad, si bien con objetivos y metodología diferentes, pero focalizados en el análisis de los manuales escolares en torno a las décadas de 1930-1960, tanto en el ámbito de la educación para la salud como en el de otros de marcada vigencia educativa, caso de la multiculturalidad y la interculturalidad (Ramírez y Ramírez, 2006; Valdemoros y Ramírez, 2007) 1 .

La motivación parte del compromiso de un equipo de investigación de salud escolar y universitaria, integrado por miembros de la Asociación Española de Medicina Escolar y Universitaria (AEMSEU) y de grupos de investigación de las Universidades Complutense de Madrid y de La Rioja. Por ello, se han puesto en marcha acciones previas tendentes a sentar las bases para esta investigación. Entre las más significativas se destaca el desarrollo y organización del IV Encuentro Internacional de Medicina y Salud Escolar y Universitaria: Los Profesionales de la Salud: Avanzando Juntos (La Rioja, 2006); el Seminario sobre Multiculturalismo e Interculturalidad en los Manuales Escolares (Berlanga de Duero, 2006); las I y II Jornadas de Salud Escolar y Universitaria (La Rioja, 2008 y 2010, respectivamente); y, por último, los Encuentros Internacionales sobre las Migraciones (Universidad de La Rioja 2008, 2009 y 2010) $)^{2}$.

El objeto de estudio del trabajo que nos ocupa se inscribe en el análisis de algunos conceptos clave, caso del de salud, higiene y educación para la salud (a partir de ahora EpS), así como de la pedagogía de la EpS y los programas de contenidos de algunos manuales escolares de las décadas 30, 40 y 50 del pasado siglo, con la pretensión de analizar y comparar el tratamiento llevado a cabo en dicha época con el efectuado en la actualidad. Asimismo, se pretende detectar algunos enfoques multiculturales e interculturales puestos de manifiesto en dichos materiales en relación a la EpS.

1. Proyectos de investigación-acción educativa que trabajan la interculturalidad: 1. "Plan Piloto de Formación Integral de Adultos para el desarrollo de la ciudad de Melilla (España)", Melilla, 1989/1992". 2. "Plan de Alfabetización y Educación Básica de Adultos de El Salvador, C.A.", San Salvador, 1993/1995. 3. Programas de Educación para la Salud y Planes Integrales del Gobierno de La Rioja. 4. Proyecto MANES (Manuales Escolares: www.uned.es/manes/). 5. Proyecto MANAL (Manuales Escolares de PANAL, Nalda (La Rioja). 6. Proyecto de Salud Bucodental (Universidad de la Rioja, AEMSEU, Universidad Complutense). Valdemoros M.: "Investigación en educación para la salud: elaboración de materiales didácticos de aula". IV Encuentro Internacional de Expertos en Salud Escolar y Universitaria: Avanzando Juntos. Logroño, 2006.

2. Las Jornadas de Salud Escolar y Universitaria, así como los Encuentros Internacionales sobre las Migraciones, pertenecen a los Programas de Extensión Universitaria del Vicerrectorado de Estudiantes de la UR, con la colaboración del Área de Didáctica de la Lengua y la Literatura (Departamento de Filologías Hispánica y Clásicas), la Oficina del Representante del Rector para la Responsabilidad Social, e instituciones, organismos, entes y asociaciones del tejido social, profesional y académico de ámbitos locales, nacionales e internacionales. También, el Seminario sobre Multiculturalismo e Interculturalidad que se celebró en el Centro Internacional de la Cultura Escolar (CEINCE), en colaboración con el Proyecto MANES, en Berlanga de Duero (Soria), España. 


\section{ESTUdiO CONCEPTUAL: SALUD, HIGIENE Y EDUCACIÓN PARA LA SALUD}

Son varios los conceptos que aparecen en el campo socio-semántico de las palabras salud e higiene. Casares (1984: 398), en la parte onomasiológica de su diccionario, recoge varios conceptos-términos de salud que la interrelacionan con otros como salubridad, sanidad, lozanía, robustez, buena disposición, euforia, eutaxia e higiene.

La higiene se asocia con algunos conceptos-términos, caso de pública, privada, alimento, bebida, fisiología, vestidura, limpieza, baño, gimnasia, deporte, juego, salud, desinfección o vacunación (Casares, 1984: 218).

Se comprobará, en adelante, cómo se trata de términos que aparecen en los manuales escolares analizados, en su referencia tanto a la salud como a la higiene.

A su vez, según el Diccionario de la Lengua Española (DRAE, 2001: 2017), salud, en su acepción primera, es el estado en el que el ser orgánico ejerce normalmente todas sus funciones; en su acepción segunda, son las condiciones físicas en que se encuentra un organismo en un momento determinado; en su acepción tercera, es libertad o bien público o particular de cada uno; en la cuarta, estado de gracia espiritual.

En cuanto a la palabra higiene, el mismo diccionario (DRAE, 2001: 1210), en su primera acepción, la define como la parte de la medicina que tiene por objeto la conservación de la salud y la prevención de enfermedades; en su acepción segunda, es limpieza, aseo de las viviendas, lugares públicos y poblaciones. Se divide en privada -aquella de cuya aplicación cuida el individuo-y pública-aquella en cuya aplicación interviene la autoridad, prescribiendo reglas preventivas-.

El concepto de salud, sin duda, ha evolucionado con el tiempo. Quizás fue la definición ofrecida por la Organización Mundial de la Salud (OMS, 1948)³) la cual consideraba la salud como un estado de completo bienestar físico, mental y social, y no solamente la ausencia de afecciones o enfermedades, la que provocó un gran salto conceptual, implicando grandes cambios para el sistema sanitario.

Años después, Dubos (1956) circunscribe la salud entre las dimensiones física y mental, si bien poniendo en evidencia el ámbito social, dado que la define como un estado físico y mental libre de dolencia que posibilita al individuo funcionar efectivamente en el ambiente en el que está ubicado.

A partir de entonces, se acentúa la tridimensionalidad de la salud, poniendo énfasis en la dimensión social de la misma. En esta línea, autores como Perea, Quintana, Murga, Limón y Corbella (1997: 30) continúan ampliando y modificando la definición de este concepto, considerándolo como el conjunto de condiciones físicas, psíquicas y sociales que permitan a la persona desarrollar y ejercer todas sus facultades en armonía y relación con su propio entorno.

Por último, Valdemoros, Ponce de León, Sanz y Ramos (2007) plantean la salud como un valor, que definen como el sentimiento de apreciación de nuestro bienestar físico, psicológico y social, que conlleva la adopción de medidas tanto preventivas como rehabilitadoras para su logro, mantenimiento y conservación.

3. http://www.who.int/es/. 
Como puede comprobarse, cada vez con mayor evidencia, se pone de manifiesto la salud en su cualidad holista. Tal es así que podría decirse que la salud se constituye en un valor transversal que engloba la práctica totalidad de virtudes humanas posibles. Así lo constata en su investigación Valdemoros (2010), verificando que la salud, en su dimensión física, engloba valores como la estética corporal o el buen estado de forma física que, obviamente, ayudarán a la dimensión psicológica de la salud al fomentar la autoestima, el autoconcepto o la superación personal, entre otras bondades; asimismo, estas cuestiones impulsarán la esfera social de la salud, optimizando las relaciones personales, la cooperación, el civismo, la solidaridad y el respeto, entre otros valores. Así pues, la salud es un triángulo cuyos vértices están en continua interrelación, guardando en su interior una importante cuantía de valores que configuran el mapa axiológico del individuo.

En otro orden de cosas, la Educación para la Salud ha supuesto en los últimos años uno de los campos de innovación y relevancia en el marco educativo de nuestro país, ganando importancia tanto en el ámbito de la educación formal como en el de la no formal.

No obstante, en sintonía con algunos autores, caso de Marías y Molina (1998), se estima que la EpS es una práctica antigua y de larga tradición, si bien ha cambiado sustancialmente su enfoque ideológico y metodológico, especialmente porque se ha evolucionado en la consideración del concepto de salud, siendo estimado en la actualidad como una cuestión global que afecta a nuestro bienestar físico, psicológico y social y no como aspecto puntual que atañe únicamente a la ausencia de enfermedad.

Otros estudiosos del tema (Perea y cols., 1997) entienden la educación como un proceso optimizador y de integración, y la salud como un estado de bienestar físico, psíquico y social. En consecuencia, definen la EpS como un proceso educativo a lo largo de la vida, encauzado tanto hacia el auto-conocimiento, en todas las dimensiones, como al ambiente que nos rodea, con el fin de tener una vida sana.

Costa y López (1998) complementan lo anterior, otorgando a la EpS la cualidad de constituirse en un proceso tanto de enseñanza-aprendizaje como de comunicación, que ha de ser planificado y sistemático, debiendo estar dirigido a facilitar la elección, el logro y la conservación de prácticas saludables, así como a dificultar aquellas que supongan riesgo.

En esta línea, Marías y Molina (1998) plantean la EpS como un proceso de formación que ha de responsabilizar al alumno para que adquiera aquellos conocimientos, actitudes y hábitos fundamentales para proteger y fomentar la salud tanto individual como colectiva, preparándolo para la adopción de un estilo de vida saludable.

Pues bien, dado que los manuales escolares se establecen en un recurso óptimo que favorece la comprensión de la educación de una etapa y su historia (Ossenbach y Somoza, 2001), en el artículo que nos ocupa se analizarán algunos de los manuales escolares españoles de las décadas de 1930, 1940 y 1950, con el fin de comprobar el tratamiento de la EpS, compararlo con el actual, rescatar aquello que se considere positivo como aportación a la actual EpS, así como suprimir lo considerado negativo o inviable en nuestros días. 
Para ello se analizarán, en primer lugar, los conceptos de Salud e Higiene y, en segunda instancia, la Pedagogía aplicada en la EpS entonces y ahora.

\subsection{Concepto de salud-higiene}

Acercándonos a la década de los 30 del pasado siglo, resulta curioso comprobar cómo prestigiosos estudiosos del momento, caso de Plá Cargol (1929: 153), destacan que la Higiene es la parte de la medicina que tiene por objeto no tan sólo la conservación de la salud sino también el perfeccionamiento del individuo y, como natural consecuencia, de la sociedad en general. Haciendo referencia a la Salud, el autor declara que ésta representa un equilibrio estable entre las actividades de nuestro cuerpo y las causas que pueden entorpecer o debilitar estas actividades; cuando este equilibrio se altera [...] surge la enfermedad. Entre estas causas destaca las inadecuadas condiciones climatológicas o de iluminación, la incorrecta alimentación o las infecciones. En este sentido, comienza a vislumbrarse una concepción no solo multidimensional de la salud (cuerpo-mente-ambiente) sino, además, una idea holista del término -al establecerse interdependencia entre las dimensiones-.

A su vez, Ascarza (1931: 64) define la Higiene como la ciencia de conservar la salud, definiendo Salud como el estado de funcionamiento normal y perfecto de nuestros órganos. Añade, en el apartado de Fisiología Humana, que enseña a conservar las funciones, pero es incapaz de evitar la muerte (Ascarza, 1931: 5). Asimismo, define Enfermedad como la perturbación de algún órgano o función y la Muerte se constituiría en la cesación de todas las funciones (Ascarza, 1931: 64). Finalmente, señala que las Funciones son muy variadas y complejas [...], y para su estudio se dividen en tres grupos, que son: de nutrición [...], de reproducción [...] y de relación, que sirven para comunicarnos con otras personas y con todo lo que es exterior a nosotros.

Ya en la década de los 40 del siglo XX continúa hablándose de que el objeto de la Higiene es enseñar lo que debemos hacer para conservar la salud. Se incide en la necesidad de observar las reglas higiénicas, porque la mayor de las desgracias es no gozar de salud (Dalmáu, 1947: 357).

De estas definiciones puede extraerse una visión de la Salud como dimensión vital que hay que cuidar y conservar a través de la Higiene, utilizando una Pedagogía de la Educación para la Salud materializada en Enciclopedias de Grado Elemental, Lecturas Científicas y Lecciones para Cursos Completos de Primera Enseñanza, escritas por reconocidos y prestigiosos autores, muy vinculados al ámbito de la Educación. Cabe destacar que en esta época ya se concibe una dimensión física de la salud (cuidado del cuerpo), una esfera socio ambiental (elementos contextuales favorecedores o perturbadores) y, por último, una mental (perfeccionamiento del individuo, equilibrio).

En los manuales analizados se realiza, igualmente, una división de la Higiene:

- Pública: la que establece reglas que deben cumplirse colectivamente e imponerse por las autoridades como la limpieza de calles, servicio de alcantarillado, surtido de aguas, cementerio, régimen de mataderos, etc. (Ascarza 1931: 64), destacándose que sin ella, enfermedades, y sobre todo las epidémicas, causarían enorme mortandad en las grandes poblaciones. En el capítulo de "La higiene en 
las ciudades", Plá (1935: 62) expresa lo siguiente: Sentid todo el respeto por estos servicios municipales: la perfección con que ellos se realicen, dirá mucho en pro de la cultura de vuestra ciudad, villa o pueblo [...] Pensad que laborando por la salud del pueblo, laboráis directamente por vuestra salud. La higiene es símbolo de cultura, por lo que se hace necesario tomar ejemplo de muchas ciudades extranjeras, en las cuales los servicios de limpieza se llevan con un cuidado esmerado y con una minuciosidad notable (Plá Cargol, 1935: 60).

- Privada: la constituyen aquellas reglas que deben cumplirse individualmente y por cada persona, como la cantidad y calidad de alimentación [...], el ejercicio físico, el aseo del cuerpo, etc., destacándose que sin higiene privada, cada persona se expone a adquirir numerosas enfermedades (Ascarza 1931: 64).

Esta división de la Higiene Ileva a considerar la Salud como algo dependiente del medio en el que se desenvuelve el individuo.

En este sentido, se detectan importantes puntos en común con el concepto de Salud de la OMS (1948), que la define como un estado completo de bienestar físico, mental y social, y no solamente la ausencia de enfermedad. Es un proceso dinámico y continuado y tiene un componente social que la relaciona con el entorno del individuo. Un entorno que no puede quedar al margen cuando se habla del mantenimiento de la salud.

Puede concluirse este apartado, pues, afirmando que tanto en los manuales antiguos como en la actualidad, el concepto de Salud tiene, de fondo, muchos elementos en común: Salud como concepto multidimensional, holista y ecológico, necesidad de una pedagogía de la educación para la salud, la Salud como una dimensión vital de la persona y de la sociedad que ha de cuidarse y conservarse, e incluso la Salud como bien más preciado, entre los muchos razonamientos que justifican su alta consideración.

\subsection{Pedagogía de la educación para la salud}

Comparando la filosofía de los años 30-40-50 del siglo XX con la actual, pueden encontrarse puntos de afinidad sobre la importancia concedida a ofrecer información rigurosa sobre el tema, sobre los factores y comportamientos de riesgo, así como a la importancia de considerar las necesidades individuales y sociales para llevar a cabo la EpS. No debe olvidarse que en dichos manuales se diferencia el tratamiento que debemos ofrecer a diferentes colectivos -bebés, niños, ancianos- y las discrepancias encontradas según ámbito geográfico -zonas rurales o urbanas-.

Del mismo modo, en la actualidad se prioriza la necesidad de individualizar la educación, en general, y la EpS, en particular, atendiendo a la diversidad y, en definitiva, adaptándose a los diferentes contextos, ámbitos geográficos, niveles socioeconómicos y culturales, así como necesidades particulares o colectivas, entre otras cuestiones.

No obstante, a pesar de las similitudes en este apartado, sí se detectan importantes matices que marcan la diferencia entre antes y ahora. Así, en los materiales de las décadas 1930, 1940 y 1950 se enseña lo que debemos hacer para conservar la salud, a través de un modelo unidireccional, transmisor de información, consejos 
y pautas, materializado en Manuales y Enciclopedias. En la actualidad, la EpS implica no sólo la transmisión de información sino, además, el fomento de la motivación, de las habilidades personales y sociales, del pensamiento crítico, de la educación en valores o del impulso de la autoestima, constituyéndose en condiciones necesarias para la adopción de medidas destinadas a la mejora de la calidad de vida. Se trata de un modelo dinámico y participativo, centrado no sólo en el saber sino, también, en el saber hacer, acorde con el enfoque por competencias, preocupado por aqueIlos conocimientos, actitudes y habilidades dirigidas a facilitar la interiorización del valor de la salud y los cambios en las conductas individuales y colectivas para conseguir determinadas metas saludables. Sin olvidar que, en último término, la decisión corresponde al individuo, por lo que el trabajo ha de centrarse en la persona y su contexto.

Realizado el estudio conceptual y poniendo de manifiesto los objetivos destacados de la EpS en los manuales analizados se procede, en el epígrafe siguiente, a la descripción de contenidos alusivos al tema.

\section{Contenidos de la EpS en los materiales didácticos de las décadas COMPRENDIDAS ENTRE 1930 Y 1950}

Realizándose una recopilación de contenidos, relacionados con la salud y la higiene, en los manuales analizados (Plá Cargol, 1929; Ascarza y Solana, 1931 y Dalmáu, 1937, 1947) puede sintetizarse el temario que se expone a continuación:

- La Higiene, su división y fundamento: nos define higiene y salud, explica la división de la higiene (pública y privada), las atenciones de la higiene, clarificando qué se entiende por temperamento y por constitución orgánica.

- Los Alimentos: clases y composición de los alimentos, recomendaciones sobre la ración alimenticia, los condimentos, cómo hacer los alimentos más asimilables, la prevención de la nocividad de algunos alimentos -cómo detectarlo, principales adulteraciones en la leche y las carnes, consecuencias de los alimentos alterados, etc.-, consejos para llevar a cabo una óptima alimentación -que sea variada, no comer en demasía, hacerlo despacio y masticando bien para facilitar la digestión-. Entre los contenidos de la EpS en la actualidad, el tema referente a la Alimentación es clave y posee muchos contenidos afines a los de entonces. Por último, se hace referencia a los grandes peligros de la época asociados a la alimentación, caso de la tenia, la triquina, el carbunco o la tuberculosis, los cuales, en la actualidad, gracias a los controles higiénicos y la inspección de los alimentos antes de su comercialización, están extinguidos; si bien, han surgido otros nuevos, como la salmonelosis, la toxoplasmosis, la colza adulterada, el mal de las vacas locas o la gripe aviar, a los que se les debe prestar atención.

- Las bebidas: el agua como bebida indispensable, su consumo, potabilidad, así como la función del agua en la higiene. Se insiste en la necesidad de ingerir agua en abundancia. Son aspectos que dietistas y profesionales de la salud en la actualidad repiten continuamente recordando que es fuente de salud. Asi- 
mismo, se habla de las bebidas alcohólicas y de alcoholismo, detallando cómo pueden tomarse para que no sean nocivas para la salud y cuáles son los efectos que produce el abuso de estas bebidas, destacándose como consecuencia del mismo la pérdida de dignidad; en la actualidad, existe consenso en que además de los riesgos físicos, deben enfatizarse aquellos personales y de relación con los otros. Además, resulta curioso la variedad de bebidas que tenemos hoy en comparación con las que entonces se ponían de manifiesto en los manuales -vino, cerveza, aguardientes, sidra y, por último, té y café como bebidas aromáticas-. También lo es el hecho de que ciertas bebidas eran prohibidas, como los aguardientes, salvo prescripción médica. Sobre el vino y la cerveza se recomienda el consumo moderado, igual que se hace en la actualidad; incluso destacan los mayores beneficios de la cerveza sobre el vino.

- El aire: su composición, efectos mecánicos y químicos, y sus peligros. Se defienden sus virtudes como purificador de la atmósfera, ofreciéndose consejos acerca de la temperatura y el grado de humedad adecuado; alertan de los peligros del hacinamiento y destacan la necesidad de cuidar el medio ambiente como fuente de salud para la población. Ya en aquella época se recomendaba el uso de vasijas de agua en los espacios con calefacción para mantener la humedad idónea; hoy se aconsejan humidificadores comercializados, más costosos y con dudosos efectos superiores a los de las vasijas de antaño. En alusión a los problemas derivados del hacinamiento es preciso denunciar que en el siglo XXI continúa siendo un grave problema para muchos colectivos desfavorecidos social y económicamente. Finalmente, se comprueba que el cuidado al medio ambiente ya era contenido fundamental de la EpS. Es cierto que en la actualidad queda lejano a la mayoría la referencia a las letrinas o las aguas encharcadas, pero es preciso denunciar los graves peligros derivados de la contaminación de las ciudades industrializadas provocada por algunas fábricas, centrales nucleares, coches, camiones, motos y otros medios de transporte contaminantes, vertederos incontrolados, etc., e incluso por desastres naturales, caso de huracanes, terremotos o lluvias torrenciales, que dejan los espacios inhabitables e imágenes desoladoras que ponen de manifiesto las condiciones infrahumanas en las que queda la población, así como los riesgos de infecciones y enfermedades.

- Las habitaciones: da consejos sobre cómo deben ser las habitaciones (amplias, secas, bien ventiladas, luminosas y limpias). Cuestión que recuerda las recomendaciones que la actual legislación educativa ofrece sobre organización de los espacios en los centros escolares. En este sentido, sería conveniente realizar una crítica acerca de que no toda la población puede disfrutar de estas condiciones en sus viviendas y escuelas.

- Vestidos, baños y ejercicio físico: presenta las materias empleadas en la fabricación de los vestidos (lino, cáñamo, algodón, lana, seda, pelo, etc.) y cómo deben emplearse según la estación del año. Transmiten que nunca deben comprimir y han de ser holgados, limpios y acomodados a la temperatura y al clima. Denuncian cómo la moda quebranta estos preceptos, cuestión igualmente presente entre las recomendaciones actuales. Defienden el ejercicio físi- 
co al aire libre como hábito necesario para tener buena salud, ya que activa todas las funciones y favorece el desarrollo. Dalmáu (1947) añade la importancia del reposo y el sueño. Son objetivos vigentes en la EpS que pretendemos en la actualidad. Puede concluirse poniendo de manifiesto una de las discrepancias detectadas entre el pasado y el presente: la variedad de tejidos con los que contamos hoy son en su gran mayoría sintéticos y poco saludables, a diferencia de los utilizados en las décadas analizadas.

- La higiene del niño: se destaca la importancia de la lactancia materna hasta los ocho-diez meses recomendando, en su defecto, leche de vaca diluida en agua, bien hervida y con un poco de azúcar. Toman el peso del niño como referente de su crecimiento y lo consideran importante para la detección de posibles problemas. Indican la importancia de la limpieza, el aire libre, el abrigo prudente y la estimulación del ejercicio. La educación en los primeros años debe limitarse al juego, ejercicios de observación y perfeccionamiento de los sentidos. Destacan el papel del médico para el tratamiento de cualquier enfermedad, evitando remedios caseros que perjudiquen. Con la salvedad de la recomendación de dar leche de vaca al niño que no puede ser amamantado -impensable en la actualidad-, el resto de consideraciones son perfectamente válidas en nuestros días. La pedagogía actual enfatiza la importancia de la educación multisensorial, la estimulación de los sentidos, el aprendizaje por descubrimiento y significativo o el constructivismo; conceptos que sentaron sus bases ya en las épocas estudiadas en el trabajo que nos ocupa.

- La higiene de los ancianos y enfermos: puede comprobarse que ya asistimos a un concepto de EpS que abarca toda la vida, desde el nacimiento hasta la vejez. Se insiste en la importancia del consejo médico. Hoy también se incide en estos aspectos.

- Enfermedades contagiosas y su higiene: se definen como enfermedades que se propagan de unas personas a otras por la ropa, el agua, los alimentos, el aire, etc., destacándose como las más frecuentes la fiebre tifoidea, la viruela, la escarlatina, la difteria, la tos ferina y la tuberculosis. Como precauciones subrayan la desinfección y aislamiento del enfermo y la necesidad de cuarentena hasta que el niño pueda volver a la escuela o el enfermo a estar en contacto con los demás. En este sentido, la filosofía actual de la EpS quizá esté más orientada a la prevención que a la curación (como sucedía entonces). Hoy resulta impensable hablar de la mayoría de las enfermedades citadas, pero han surgido otras nuevas no menos graves, caso de la gripe A, el VIH/SIDA y el resto de enfermedades de transmisión sexual. Del mismo modo, queda lejos el aislamiento como modo de prevenir contagios. A pesar de ello, no debemos olvidar que en pleno siglo XXI quedan muchos países y zonas azotadas por enfermedades contagiosas que deberían ser erradicadas.

Finalmente, Dalmáu (1937, 1947), en ambas ediciones, incrementa los contenidos expuestos con otros dos: Higiene de las estaciones, que ofrece consejos para que los cambios estacionales no perjudiquen nuestra salud; e Higiene de los sentidos, la cual ha de llevarse a cabo a través de la limpieza y la no exigencia de un tra- 
bajo excesivo. Puede intuirse que el segundo contenido guarda relación con la prevención del hoy usualmente aludido estrés.

Resulta curioso el hecho de que la salud buco-dental no forme parte del programa de contenidos de los manuales escolares analizados, cuando en la actualidad es uno de los asuntos prioritarios desde los niveles iniciales del sistema educativo.

En definitiva, puede apreciarse cómo los contenidos que hoy figuran en la mayoría de los programas de EpS están dotados de nuevos matices que surgen, en numerosas ocasiones, fruto de las nuevas necesidades acaecidas en nuestra sociedad, si bien continúan manteniendo muchos puntos de contacto con los trabajados en los programas de los manuales escolares examinados. Así, en la actualidad podemos encontrar, entre otros, los siguientes contenidos:

- Educación para la Salud y el Consumo. Concepto e implicaciones curriculares.

- Principales problemas de salud en el medio escolar.

- La higiene. Prevención de enfermedades y accidentes. Control de la salud escolar.

- Salud buco-dental.

- Medio ambiente.

- Prevención de drogodependencias.

- Sexualidad y afectividad. Prevención de riesgos asociados.

- Alimentación y nutrición.

- Acoso escolar. Problemas de convivencia. Bullying.

- Educación para el consumo. La publicidad.

- Los juguetes: sus tipos. Normas de seguridad. La publicidad de los juguetes.

Se comprueban, en la actualidad, más cambios de forma que de fondo: nuevas nomenclaturas para idénticos contenidos, si bien se añaden otros referentes a la publicidad, el consumo y los problemas de convivencia. Se trata de una realidad propia de la idiosincrasia de nuestra época, que en las décadas analizadas no era necesario abordar por su inexistencia. Cabe destacar, además, que los problemas en la convivencia que se detectan en el presente se ven agudizados por los cambios vertiginosos que acontecen en nuestra sociedad y los entornos multiculturales que no siempre dan como fruto la anhelada interculturalidad, los cuales requieren ser tratados con urgencia. En este sentido, la EpS puede y debe constituirse en escenario pedagógico por excelencia que brinde atención a estos problemas.

\section{Modelos materiales didÁcticos POSibles}

\subsection{Modelos}

La EpS puede llevarse a cabo desde modelos diversos. A continuación se ponen de manifiesto los más habituales.

- Transversalidad: en las diferentes asignaturas impartidas al alumnado, aprovechando en cada una de ellas los momentos idóneos para el tratamiento de los diferentes contenidos. 
- Puntualmente, en las distintas áreas y asignaturas de la educación infantil, primaria y secundaria. Para ello es necesaria la buena disposición del profesor, la formación en esta materia y la creencia de que con ello no se le "resta tiempo" a su asignatura, sino que lo gana.

- Programas de diferentes instituciones de la Comunidad que trabajan la EpS en los Centros Educativos a través de profesionales externos a los mismos y en actuaciones planificadas, pero puntuales. A modo de ejemplo, en La Rioja (España) las encontramos en los múltiples programas para la población de Educación Secundaria Obligatoria, de los que es responsable la Consejería de Salud -Prevención de Embarazos no deseados y SIDA, Educación en valores a través del cine, Prevención del consumo de alcohol, tabaco y otras drogas, Salud bucodental escolar, o programa de Niño sano-; el Programa de prevención de riesgos en la infancia, del que es responsable la Consejería de Industria, Innovación y Empleo; el Programa de Buenos Tratos, con el que se implica la Consejería de Servicios Sociales; el Programa para la convivencia y prevención del acoso escolar, favorecido por la Consejería de Educación, Cultura y Deporte. Además, se cuenta con diversas experiencias dependientes del Ayuntamiento de la capital (Logroño) y otros de la provincia, que velan por la prevención de drogodependencias, el trabajo en habilidades sociales o la educación sexual y afectiva, entre los múltiples temas que se abarcan. Otras entidades, caso del Comité Ciudadano AntiSida, ALCER (Asociación de Lucha contra las Enfermedades Renales), Cruz Roja o diferentes asociaciones socioculturales ofrecen múltiples actividades focalizadas en la EpS.

\subsection{Materiales didácticos}

- Editoriales: libros, cuadernos y carpetas de actividades, material audiovisual y software, entre otros.

- Instituciones: dípticos, trípticos, folletos divulgativos, cuadernos didácticos para el alumnado, la familia y el profesorado, etc.

- Fabricantes: manuales de uso, folletos, publicidad pedagógica, juegos, cuadernillos, etc.

- De elaboración educativa propia: cuentos en acordeón y cuadernos-libro elaborados artesanalmente, que tienen como eje vertebrador alguno de los temas clave en EpS (Valdemoros, 2005) y en multi e interculturalidad (Ramírez y Ramírez, 2006; Valdemoros y Ramírez, 2007).

\section{Algunos aspectos de multiculturalidad e interculturalidad en los ENFOQUES DE ESTOS MANUALES}

Se parte de que estos manuales sólo estaban al alcance de aquellos colectivos más favorecidos social, económica y culturalmente. La escolarización no era algo generalizado y menos en zonas rurales en las que los niños trabajaban lo antes posible para ayudar a la economía familiar. Algo similar podemos apuntar en la actualidad; la EpS no siempre se Ileva a cabo de modo integral. En muchas ocasiones, se 
realizan actuaciones aisladas que informan sobre algunos aspectos, pero no educan por y para la salud.

Antes y ahora se detectan diferencias en las oportunidades higiénicas entre diferentes grupos sociales. Entonces, la división era urbana/rural. Generalmente, en los pueblos las oportunidades de educación para la higiene eran menores y las condiciones de vida, también. De igual modo, las diferencias entre pobres y ricos eran significativas en este aspecto. Hoy no se está libre de estas diferencias. Se descubren grupos muy desfavorecidos socialmente que viven en unas condiciones higiénicas que brillan por su ausencia, caso de algunos poblados gitanos o ciertos colectivos de inmigrantes que llegan "con lo puesto", viéndose obligados a vivir hacinados.

La necesidad de convivencia entre culturas, en la actualidad, obliga a continuas adaptaciones en hábitos de higiene: alimentación, enfermedades, costumbres sociales y culturales, entre otros aspectos, siendo obligación de todos asumir normas y pautas que no pongan en peligro nuestra salud ni la de los demás.

\section{Conclusiones}

Fruto del análisis comparativo llevado a cabo en el estudio que nos ocupa, pueden extraerse las siguientes conclusiones:

1. En las décadas 1930, 1940 y 1950 ya se evidencia una preocupación pedagógica por la higiene y la educación para la salud.

2. Se constatan más cambios en la forma que en el fondo, dado que en ambas épocas se mantienen muchos de los contenidos fundamentales, si bien difiere el léxico utilizado.

3. Se comprueban cambios en la pedagogía y la didáctica de la EpS. Antes destacaba un modelo unidireccional, pasivo y transmisor de información; ahora se departe acerca de un modelo participativo, bidireccional, en el que no sólo interviene el saber, sino también el saber hacer (conocimientos, actitudes y procedimientos). Si bien, se debe aclarar que no siempre en la práctica es así.

4. Se descubre que la filosofía actual de la EpS está más orientada a la prevención que a la curación, a diferencia de lo que ocurría en tiempos pasados.

5. La aparición de nuevas necesidades modifican los programas de contenidos de EpS. Hoy se hace preciso abordar materias relacionadas con el VIH/SIDA, los medios de comunicación y la publicidad, los problemas de convivencia, los riesgos de las nuevas tecnologías o la esclavitud de las modas, entre otras.

6. En las prácticas saludables de antaño se localizan hitos plenamente vigentes y útiles. No se ha inventado todo en el presente, sino que en aquellos tiempos se sentaron muchas bases que deben ser valoradas y tenidas en consideración.

7. En los manuales analizados se comprueba el alto nivel de conciencia, al menos pedagógica, que tenían sobre la higiene y la salud. La salud es el bien más preciado y la conseguimos a través de una educación de la higiene adecuada, hoy EpS.

8. Antes y ahora se siguen descubriendo grupos humanos desfavorecidos con inferiores oportunidades de higiene y, por tanto, de salud. 
En síntesis, se pone de manifiesto una posibilidad real de enriquecimiento en el ámbito que nos ocupa, a través del intercambio y la recuperación de prácticas preventivas y sanitarias de otras culturas y de otros tiempos, erróneamente descalificadas, expresadas en materiales didácticos básicos, como fueron los manuales escolares. La EpS puede constituirse en ámbito de gran importancia para cubrir las necesidades de salud en poblaciones desplazadas, fuera de su marco cultural. Se hace preciso, pues, seguir potenciando proyectos de EpS, así como conocer científicamente estos procesos.

\section{REFERENCIAS BIBLIOGRÁFICAS}

ASCARZA, V. y SOLANA, E. (1931). Fisiología e higiene. Madrid: El Magisterio Español.

CASARES, J. (1994). Diccionario ideológico de la lengua española. Barcelona: Gil y Gaya, S.A.

COSTA, M. y LÓPEZ, E. (1998). Educación para la Salud: Una estrategia para cambiar estilos de vida. Madrid: Pirámide.

DALMÁU, C. 1937 (1922). Enciclopedia Cíclico-Pedagógica. Gerona-Madrid: Dalmáu Carles, Pla. E. C.

DUBOS, R. (1956). El espejismo de la salud: utopías, progreso y cambio biológico. México: Fondo de Cultura Económica.

MARÍAS, I. y MOLINA, M. C. (1998). Educación para la Salud. Recursos para educadores. [Disponible en: http://www.xtec.cat/ imarias/].

ORGANIZACIÓN MUNDIAL DE LA SALUD Y LOS OBJETIVOS DE DESARROLLO DEL MILENIO. [Disponible en http://www.who.int./mdg/es/].

OSSENBACH, G. y SOMOZA, M. (2001). Los manuales escolares como fuente para la historia de la educación en América Latina. Madrid: UNED/Manes.

PEREA, R.; QUINTANA, J. M.; MURGA, M. A.; LIMÓN, M. R. y CORBELLA, M. (1997). Curso de Educación para la Salud: Fundamentos y justificación de la Educación para la Salud. Madrid: UNED-FUE.

PLÁ CARGOL, J. (1929). Elementos de organografía. Fisiología e higiene. Gerona: Dalmáu Carles, Pla, S.A. Editores.

—, (1935). Otras lecciones de cosas. Gerona-Madrid: Dalmáu Carles, Pla, S.A. Editores.

RAMÍREZ, J. y RAMÍREZ, R. (2006). Textos para la interculturalidad. En M. Casado, R. González y M. V. Romero (coord.) (pp.2341-2352). Análisis del discurso: lengua, cultura y valores: Actas del I Congreso Internacional, Vol. 2. Madrid: Arco Libros, S. L.

REAL ACADEMIA ESPAÑOLA (1992). Diccionario de la lengua española. Madrid: Real Academia Española.

VALDEMOROS, M. A. (2005). Investigación en didáctica de la lengua y la literatura escritas: elaboración de materiales didácticos escritos de aula sobre educación para la salud. En J. Ramírez (coord.). La lengua escrita (pp. 835-842). Barcelona: SEDDL-Universidad de Barcelona. 
VALDEMOROS, M. A. (2010). Los valores en el ocio físico-deportivo. Análisis y propuestas educativas. Logroño: Universidad de La Rioja.

VALDEMOROS, M. A.; PONCE DE LEÓN, A.; SANZ, E. y RAMOS, R. (2007). El valor de la salud en las experiencias de ocio físico-deportivo de jóvenes y adolescentes: conceptualización y estado de la cuestión. Contextos Educativos. Revista de Educación, 10, 117-132.

VALDEMOROS, M. A. y RAMÍREZ, J. (2007). La multiculturalidad en algunos manuales escolares de las décadas de los años 1930-1960. En J. Giró (coord.). La escuela del siglo XXI [Recurso electrónico]: la educación en un tiempo de cambio social acelerado. Logroño: Universidad de La Rioja. [Disponible en: http:// dialnet.unirioja.es/servlet/articulo?codigo=2376228]. 